This document is the Accepted Manuscript version of a Published Work that appeared in final form in Journal of Materials Chemistry C 7(22) : 6548-6558 (2019), copyright (C) 2019 The Royal Society of Chemistry. To access the final edited and published work see https://doi.org/10.1039/c9tc01609k

\title{
How cyclic chain topology can reduce the crystallization rate of Poly(3- hexylthiophene) and promote the formation of liquid crystalline phases in comparison with linear analogue chains
}

\author{
Jon Maiz ${ }^{a}$, Guoming Liu ${ }^{b}$, Fernando Ruipérez ${ }^{a}$, Nicolas Delbosc ${ }^{c}$, Olivier Coulembier ${ }^{c}$, Dujin Wang $^{b}$, \\ Alejandro J. Müller*a,d

\begin{abstract}
${ }^{a}$ POLYMAT and Polymer Science and Technology Department, University of the Basque Country UPV/EHU, Paseo Manuel de Lardizabal 3, 20018 Donostia-San Sebastián, Spain. Email: jon.maiz@polymat.eu, alejandrojesus.muller@ehu.es

${ }^{\mathrm{b}}$ Beijing National Laboratory for Molecular Sciences, CAS Key Laboratory of Engineering Plastics, CAS Research/Education Center for Excellence in Molecular Sciences, Institute of Chemistry, Chinese Academy of Sciences, Beijing 100190, P. R. China.

'Laboratory of Polymeric and Composite Materials, Center of Innovation and Research in Materials and Polymers (CIRMAP), University of Mons, Avenue Maistriau 19, B-7000 Mons, Belgium

'IKERBASQUE, Basque Foundation for Science, Bilbao, Spain.

Electronic Supplementary Information (ESI) available: See DOI: 10.1039/x0xx00000x
\end{abstract}

We have studied how cyclic topology affects the crystallization and morphology of $\pi$-conjugated poly(3hexylthiophene) ( $\mathrm{P} 3 \mathrm{HT}$ ) molecules by comparing linear and cyclic analogues for the first time, with three different chain lengths. We employ a range of experimental techniques including wide-angle $\mathrm{X}$-ray scattering (WAXS), polarized light optical microscopy (PLOM), differential scanning calorimetry (DSC) and a combination of Polarized Light Optical Microscopy (PLOM) and Spectroscopy (S). The crystallization and melting points, melting/crystallization enthalpies, and overall crystallization kinetics of cyclic P3HTs are found to be substantially lower than their linear counterparts. The results are explained by the higher rigidity of cyclic molecules, as predicted by density functional theory (DFT) calculations, in the low molecular weight range explored here, in comparison with linear P3HT chains. Additionally, we have found that cyclic P3HT can form liquid crystalline phases above their crystalline melting points and a new crystal form at lower temperatures. PLOM, WAXS, DSC and PLOM/S results indicate that cyclic P3HTs display bipolar and concentric nematic textures that disappear at a well-defined nematic-isotropic transition temperature. Such liquid crystalline textures are completely absent in the linear P3HTs analogues studied here. We conclude that the cyclic topology induces the formation of liquid crystalline phases as the rigid P3HT oligomers can self-assemble above their crystalline melting temperatures.

\section{Introduction}

Cyclic polymers show exceptional properties due to the nonexistence of chain ends and compact coil conformations, in comparison with linear polymers. Different physical properties such as crystallinity, mechanical strength, and conductivity, are affected by chain topology. The influence of the cyclic chain topology on polymer crystallization has been recently reviewed. ${ }^{2}$ A number of crystallization studies of linear and cyclic polymers have been performed and different results have been obtained in the literature. For that reason, a universal explanation for their properties is not always possible and it seems that the type of material under study plays a large role on the crystallization of cyclic chains, apart from other factors such as different levels of purity. ${ }^{2}$

In the reported literature different trends can be found:

(1) Cyclic polymer exhibit larger crystallization $\left(T_{c}\right)$ and melting temperatures $\left(T_{m}\right)$, faster spherulitic growth rates $(G)$ and/or overall crystallization rates $\left(1 / \tau_{50 \%}\right)$ in comparison with linear polymers. Such trends have been observed for Poly $\left(\varepsilon\right.$-caprolactone) $(P C L)^{3}$ and Poly(lactide) $(P L A)^{3-7}$, which are some of the most studied cyclic polymers. The results have been explained by thermodynamic and kinetic arguments. Equilibrium melting points $\left(T_{m}{ }^{\circ}\right)$ of the cyclic polymers have been found to be higher than those of linear polymer analogues, for entropic reasons. The kinetic reasons for faster crystallization in cyclic polymers are the higher diffusion rates of cyclic chains due to their more compact coil conformations and lower entanglement densities. These tendencies can be affected by the purity of the samples, since it has been shown that impurities of linear chains cause threading effects (i.e., linear molecules connect cyclic chains by threading through them creating long-lived entanglements) leading to lower diffusion rates and in consequence lower crystallization rates. 
(2) Other types of polymers have been reported to behave differently. These other cyclic polymers have higher nucleation density, lower $T_{m}{ }^{\circ}$, and lower $G$ than linear analogues of similar molecular weights. This group include poly(tetrahydrofurans) $(\mathrm{PTHFs})^{8,9}$ Until now the reasons for the different behaviors are unknown and it is considered that each polymer must be studied separately in order to determine the type of behavior that it presents. In all previous works, the study of chain topology on crystallization has been carried out in flexible polymers with simple unsubstituted linear repeating units ${ }^{1}$ (i.e., PCL, PLAs, PEs, PEOs, PTHF).

Fully conjugated macrocycles can be considered infinite $\pi$-conjugated systems (with altered electronic properties) leading to different final properties as compared to their linear analogues. ${ }^{10,11}$ These polymers should have several applications: in OPVs, OFETs, and OLEDs ${ }^{11,12}$ because of: (i) their twisted or bent structure should help intermolecular contacts and charge transport; (ii) they lack end groups (that create defects in linear polymers), so they do not act as trap-sites for charges; (iii) they frequently have an altered electronic structure; and (iv) they have a defined cavity that can be a host for electronically useful guest molecules. ${ }^{11}$ In each device, performance is strongly associated with polymer morphology and the applications are also linked with the formation of stable and soft liquid-crystalline materials.

Liquid crystalline (LC) mesophases of semiconducting polymers are examples of thermodynamically stable liquid states with higher degree of molecular order. ${ }^{13} 14$

In this article, we have found that cyclic P3HT can form a nematic phase, as we will demonstrate below. Nematic LCs are characterized by their director field, $n^{*}$, which can be visualized by using polarized light optical microscopy (PLOM). When the system is confined, usually to a spherical shell, $\boldsymbol{n}^{*}$ is essentially broken by the presence of topological defects. These particular systems are called polymer dispersed liquid crystals (PDLCS). In general, PDLCs are micrometer-sized birefringent liquid crystalline droplets, usually dispersed in an optically transparent and uniform polymer matrix and have spatially varying refractive index and efficient light-scattering properties. In this kind of systems, the confinement effect dominates over the bulk properties of liquid crystals. ${ }^{15}$

One of the most studied conjugated polymers is poly(3-hexylthiophene) (P3HT). ${ }^{16}$ Hence, it is an ideal polymer to study the influence of chain topology, when the material is synthesized in a cyclic chain topology. In this work, the thermal and structural properties of cyclic and linear P3HT were investigated as a function of chain length. Remarkable changes in behavior are triggered by the lack of chain ends and we report for the first time the formation of a LC nematic phase in cyclic P3HT promoted by the enhanced ring rigidity as compared to its linear analogue, as well as a new crystal phase at lower temperatures.

\section{Experimental section}

\section{Materials and Methods}

Experiments were performed on linear and cyclic poly(3-hexylthiophene) (P3HT) polymers of different chain lengths. Cyclic P3HT have been prepared by intramolecular McMurry coupling reaction, as previously published by some of us. ${ }^{17}$

In the present work, 3 linear-P3HT (I-P3HT) samples and 3 cyclic-P3HT (c-P3HT) samples were studied with average DP values of 18,25 and 42. These values correspond to molar masses values $\left(M_{n}\right)$ of $3.8,5.1$ and $11.8 \mathrm{kDa}$, respectively. The samples are named $/ 18,125$ and $/ 42$ for linear samples; and $c 18, c 25$ and $c 42$ for cyclic ones.

As explained above, the crystallization of cyclic chains depends on the polymer nature, and on the level of purity, among other factors. To date, except in very peculiar conditions, the preparation of pure macrocycles is always accompanied by the presence of a few poisoning impurities, such as linear chains or longer cycles, as was observed recently in PLLA and PCL cyclic samples in the literature estimating a level of impurity close to $15 \mathrm{~mol} \%$ depending on the molecular weight. ${ }^{2,4}$ In this work, Size Exclusion Chromatography (SEC) analysis and MALDI-ToF analysis are performed and the results are shown in the Supporting Information section (Figure S1 and S2). The linear contamination of our samples is around 5, 10 and $16 \%$ for $c 18, c 25$ and $c 42$ samples, respectively.

\section{Characterization}

\section{Differential Scanning Calorimetry (DSC)}

A PerkinElmer 8000 DSC equipped with an Intracooler II was employed. All the experiments were performed under ultrapure nitrogen flow, and the instrument was calibrated with indium and tin standards. Samples of $5 \mathrm{mg}$ were used. First, non-isothermal scans were performed, where the samples 
were melted for $3 \mathrm{~min}$ at temperatures high enough to erase any previous thermal history. Then a cooling scan at $20 \stackrel{\circ}{\circ} / \mathrm{min}$ was recorded, followed by a subsequent heating scan, also at $20^{\circ} \mathrm{C} / \mathrm{min}$, from room temperature (RT) to the final temperatures given by the melting temperatures for each sample.

Isothermal crystallization experiments were performed by the procedure recommended by Lorenzo et al. ${ }^{1}$, in order to determine the overall crystallization rate of the samples (i.e., including both nucleation and growth) by DSC. Samples were heated to $30 \stackrel{\circ}{\circ}$ above their corresponding peak melting temperatures and kept at that temperature for $3 \mathrm{~min}$ to erase previous thermal history. Then the samples were rapidly cooled at $60 \mathrm{o} / \mathrm{min}$ to the chosen isothermal crystallization temperature $\left(T_{c}\right)$. The $T_{c}$ range employed for each sample was previously determined by preliminary tests to ensure that no crystallization occurred during the cooling step. ${ }^{8}$

\section{Wide Angle X-Ray Scattering (WAXS)}

WAXS measurements were carried out on a Xeuss 2.0 SAXS/WAXS system (Xenocs SA, France). A Cu K $\alpha$ Xray source (GeniX3D Cu ULD), generated at $50 \mathrm{kV}$ and $0.6 \mathrm{~mA}$, was utilized to produce X-ray radiation with a wavelength of $1.5418 \AA$ A. A semiconductor detector (Pilatus 300K, DECTRIS, Swiss) with a resolution of $487 \times 619$ pixels (pixel size $=172 \times 172 \mu \mathrm{m} 2$ ) was used to collect the scattering signals. The one-dimensional intensity profiles were integrated from background corrected 2D WAXS patterns. The temperature of the samples was controlled by a Linkam THMS600 hotstage.

\section{Polarized Light Optical Microscopy (PLOM)}

The superstructural semi-crystalline morphology of polythiophene samples was examined with an OLYMPUS BX51 polarized light microscope connected to an OLYMPUS SC50 camera and a Mettler FP82HT hot stage provided with a liquid nitrogen flow. Samples were placed between glass covers and heated $30{ }^{\circ} \mathrm{C}$ above their melting temperature $\left(T_{m}\right)$ (given by the melting peak observed by DSC), and kept at this temperature for $5 \mathrm{~min}$ in order to erase their thermal history. Samples were then cooled at $5{ }^{\circ} \mathrm{C} \cdot \mathrm{min}^{-1}$ to a temperature at which $\mathrm{P} 3 \mathrm{HT}$ crystals started to appear. In $c$ - $\mathrm{P} 3 \mathrm{HT}$ samples, we found that different morphologies appeared at temperatures higher than the crystalline melting temperature. The appearance of these different morphologies, phases and transitions will be explained in the results and discussion section.

The observed new transitions were studied by a combination of Polarized Light Optical Microscopy and Spectroscopy techniques. Recently, Martin et al. ${ }^{18}$ experimentally determined the isotropic-nematic (IN) transition, $T_{\text {IN }}$, of PFO samples and P3EHT-181 using a method based on polarized light optical microscopy and spectroscopy (PLOM-S). All the experimental procedure and the samples preparation are wellexplained in reference 18. An Olimpus BX51 microscope and Ocean Optics USB2000+ spectrometer are used to conduct these experiments.

The samples used in these experiments were the same ones used before in the morphological studies by PLOM technique and in order to determine the mesophase transitions the samples were cooled as we have explained in the PLOM experimental section. A cooling rate of $1{ }^{\circ} \mathrm{C} / \mathrm{min}$ was applied in this case and the experiments were programmed to acquire a spectrum every $0.5{ }^{\circ} \mathrm{C}$. The transmitted intensity at each temperature was then integrated between the suitable wavelength range $(580$ to $700 \mathrm{~nm})$ to obtain the value of the total intensity transmitted through the materials.

Quantum chemical calculations.

Density functional theory (DFT) calculations have been performed with the Gaussian 16 suite of programs. ${ }^{19}$ Structure optimization was carried out in gas phase using the B3LYP functional ${ }^{20,21}$ and the 6$31+G(d)$ basis set. $^{22}$

\section{Results and discussion}

Figure 1 shows standard crystallization and melting behavior of linear and cyclic P3HT with average DP values of 18, 25 and 42. Figure 1a shows that in all cases cyclic samples have lower crystallization temperatures $\left(T_{c}\right)$ and melting temperatures $\left(T_{m}\right)$ than their linear analogues. Moreover, cyclic polymers present a different behavior in comparison to the linear analogues, since they exhibit two exotherms and endotherms in the temperature range between 100 and $200 \stackrel{\circ}{\circ}$ that are difficult to observe because of their low enthalpy values. With the aim to check and study the origin of these peaks, P3HT samples were examined by means of PLOM-S and X-Ray Diffraction. 
The relevant parameters obtained from the DSC results (Figure 1) are presented in Figure 2.
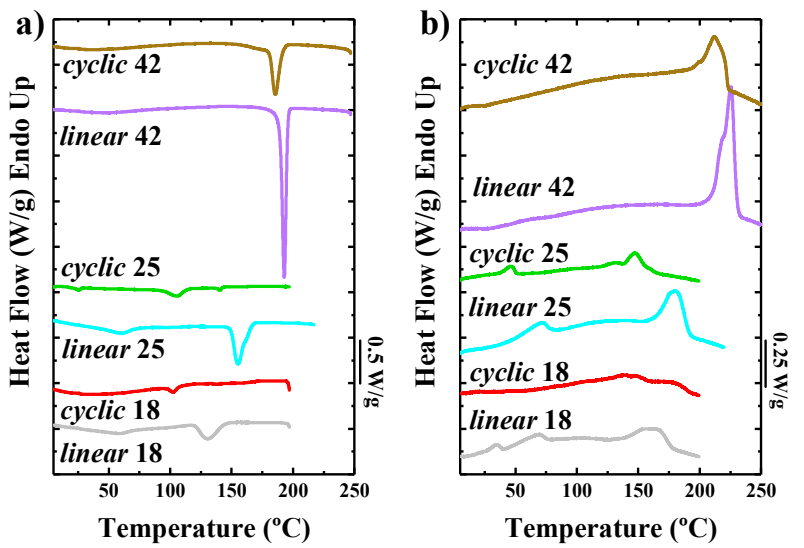

Figure 1. a) Cooling DSC scans from the melt and b) subsequent heating scans for the linear $(I)$ and cyclic (c) samples with DP of 18,25 and 42 . Scanning rate: $20^{\circ} \mathrm{C} / \mathrm{min}$.

The increase in P3HT molecular weight induces an increase in both $T_{c}$ and crystallization enthalpy $\left(\Delta H_{c}\right)$. The values of $T_{c}$ and $T_{m}$ for the cyclic polymers are lower as compared to those of the corresponding linear samples (as DP decreases, the difference between linear and cyclic samples increases). Moreover, the enthalpy values of the cyclic polymers are much lower than their linear homologues, indicating a decrease in crystallinity degree when the chains have a ring topology.

In the particular case of crystallization, the literature reports several trends and a general interpretation for all cyclic polymers is not possible. The reported trends in the literature depend on the type of material used and recently, different theoretical and experimental works have been published. ${ }^{2,4,23-26}$
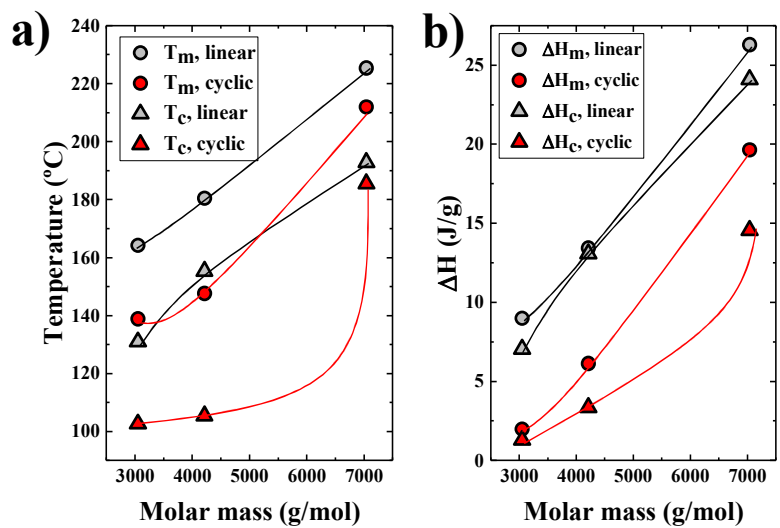

Figure 2. a) Experimental melting temperatures (circles) and crystallization temperatures (triangles) for $I$ P3HT (grey symbols) and for $c$ - $\mathrm{P} 3 \mathrm{HT}$ (red symbols) as a function of molar mass and b) experimental melting enthalpy (circles) and crystallization enthalpy (triangles) for I-P3HT (grey symbols) and for c-P3HT (red symbols) as a function of molar mass.

In our case, as $\mathrm{P} 3 \mathrm{HT}$ is a rigid polymer, oligomeric cycles probably have a more rigid chain conformation (as confirmed by simulations presented below) as compared to linear analogues, thereby making more difficult the crystallization process, leading to lower crystallization and melting temperatures and lower crystallinity degrees. It is also important to mention that the cyclic P3HT samples employed here are not pure and contain different amounts of linear chains and larger P3HT cycles (see Supplementary Information, Figure S1 and S2). However, as P3HT molecules are bulky and rigid, we speculate that it is unlikely that a threading effect similar to that found in mixtures of flexible linear and cyclic polymeric molecules ${ }^{2,7}$ is present here. We have performed isothermal crystallization experiments in the DSC to further compare the crystallization rates of linear and cyclic P3HT. Figure 3 shows the representation of several kinetic parameters of crystallization as a function of the crystallization temperature. The inverse of the half-crystallization time, determined by means of DSC isothermal crystallization experiments, is an experimental measure of the 
overall crystallization rate that includes both nucleation and growth contributions. In Figure 3a, the inverse of the half crystallization experimental data is plotted with fitting to the Lauritzen and Hoffman theory. In Figure $4 \mathrm{~b}$ the final value of the isothermal crystallization enthalpy (after the crystallization has saturated) is plotted as a function of crystallization temperature.

The results presented in Figure 3 are fully consistent with the non-isothermal DSC measurements shown in Figures 1 and 2 . As $1 / \tau_{50 \%}$ versus $T_{c}$ curves shift to lower temperatures, this implies that the sample needs a larger supercooling for crystallization, and if the curves are extrapolated in a wide $T_{c}$ range, the results also imply a crystallization rate reduction. As the crystallization rate reduces, the degree of crystallinity achieved is also seen to reduce.
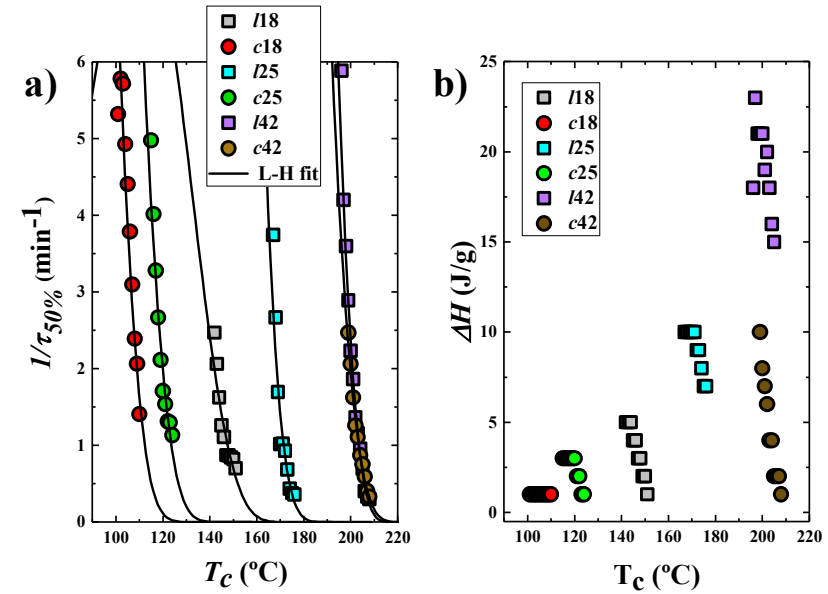

Figure 3. a) Inverse of half crystallization times as a function of $T_{c}$ and b) enthalpy values during isothermal crystallization process as a function of $T_{c}$.

The data in Figure 3 indicates that cyclic chains crystallize slower than linear chains, however the difference in crystallization rate between cyclic and linear chains reduces as the DP or molecular weight increases. Increasing the size of cyclic $\mathrm{P} 3 \mathrm{HT}$ probably releases conformational strain and the molecule resembles more its linear analogue.

In the narrow and low molecular weight range explored here, linear and cyclic P3HT molecules increase their crystallization rate as the DP value increases. In the oligomeric regime, the nucleation probability usually increases with chain length, as very small chains have difficulty in forming stable nuclei. ${ }^{27}$

The large reduction in crystallization enthalpy under isothermal conditions is remarkable, and it also demonstrates the topological constraints faced by the more rigid cyclic molecules to pack into crystals. The maximum crystallization enthalpy achieved reduces as DP decreases in cyclic P3HT by a factor of 14 when comparing cyclic samples with the maximum and the minimum DP values. In the case of their linear analogues, the reduction in the maximum crystallization enthalpy achieved is only a factor of 4 . Therefore, even though the DP values have an important effect on the crystallization rate of linear $\mathrm{P} 3 \mathrm{HT}$, chain topology has an even larger effect in the molecular weight range explored here.

The isothermal experimental data obtained from the DSC measurements were fitted to the Avrami equation following the procedure proposed by Lorenzo et al. ${ }^{1}$ The Avrami equation can describe the primary crystallization process in polymers. ${ }^{28,29}$ The equation can be written in the following form, ${ }^{1}$

$$
1-V_{c}\left(t-t_{0}\right)=\exp \left(-k\left(t-t_{0}\right)^{n}\right)
$$

This particular arrangement of the equation takes into account the role of the induction time, $t_{0}$, in the fitting. The other parameters are the relative volumetric transformed fraction $V_{c}$, the overall crystallization rate constant, $k$ (that includes both contributions of the crystallization process: nucleation and growth), and the Avrami index, $n$, which is related to the time dependence of the nucleation and the crystal growth geometry. In order to calculate these parameters, isothermal crystallization experiments are performed (supporting information, Figure S3 and Figure S4).

Figure 4 shows one example of the good agreement between the Avrami model and the experimental data in the primary crystallization regime (up to about $50 \%$ conversion or the peak value of the crystallization 
exotherm). The Avrami index obtained for the 142-P3HT sample was 2.7, which can be approximated to 3. This indicates that the material is crystallizing in instantaneously nucleated spherulites.

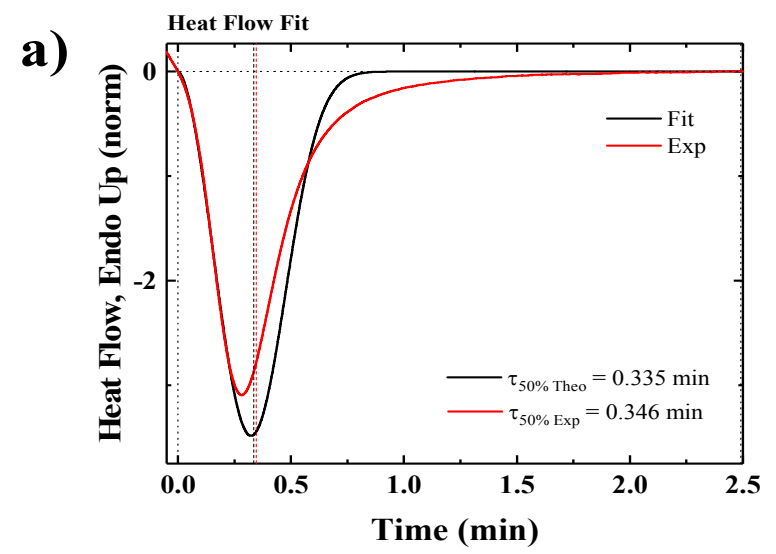

b)

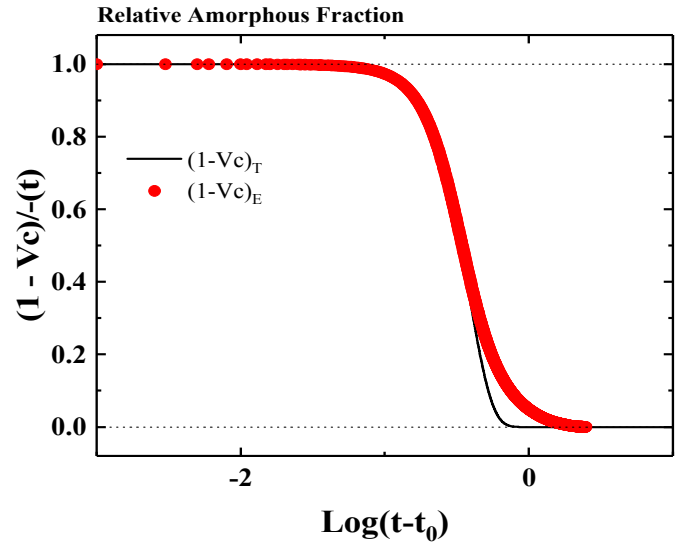

c)

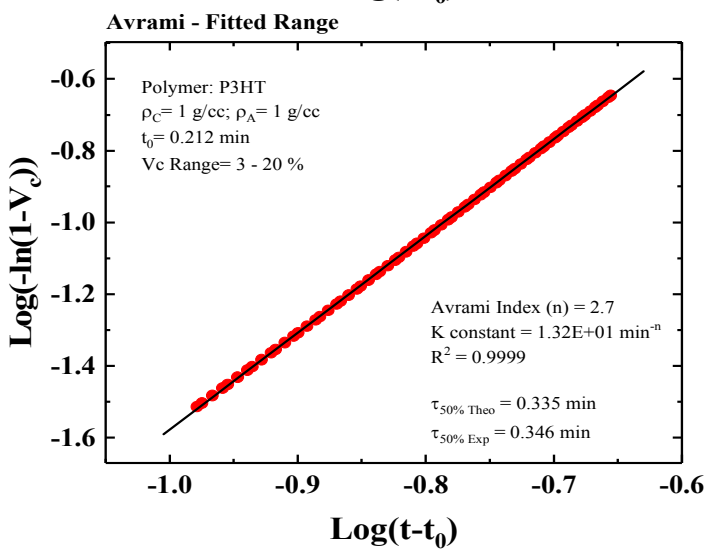

Figure 4. $(a-c)$ The fit to the Avrami equation using the Origin plug-in developed by Lorenzo et al. ${ }^{1}$ as compared to the DSC experimental data obtained for the I42-P3HT sample.

Figure 4a shows a comparison between the experimental and the predicted DSC isothermal scans. Figure 4c shows that the Avrami equation can perfectly describe the overall crystallization kinetics of $\mathrm{P} 3 \mathrm{HT}$ in the primary crystallization range (with a correlation coefficient of 0.9999), before the spherulites impinge on one another (i.e., free growth regime), in a conversion range up to $25 \%$. The fit is also quite good for the particular case of $\mathrm{P} 3 \mathrm{HT}$ up to much higher conversion (at least up to $50 \%$ as judged by the agreement between the predicted and the experimental half-crystallization times, also shown in the captions within the figure), as shown in Figures $4 a$ and $b$.

The isothermal rate constant $k$ has units that depend on $n$ (i.e., $\min ^{-n}$ ), and since $n$ values are not constant, a direct comparison cannot be made. One way to overcome this difficulty is to elevate $k$ to $1 / n$, so that all values of $k$ are expressed in reciprocal time units (in this case $\min ^{-1}$ ). Figure 5a shows how the $k^{1 / n}$ values 
change with $T_{c}$. Moreover, the Avrami index values, obtained from fitting P3HT isothermal crystallization data, are plotted in Figure $5 \mathrm{~b}$ against the crystallization temperature.
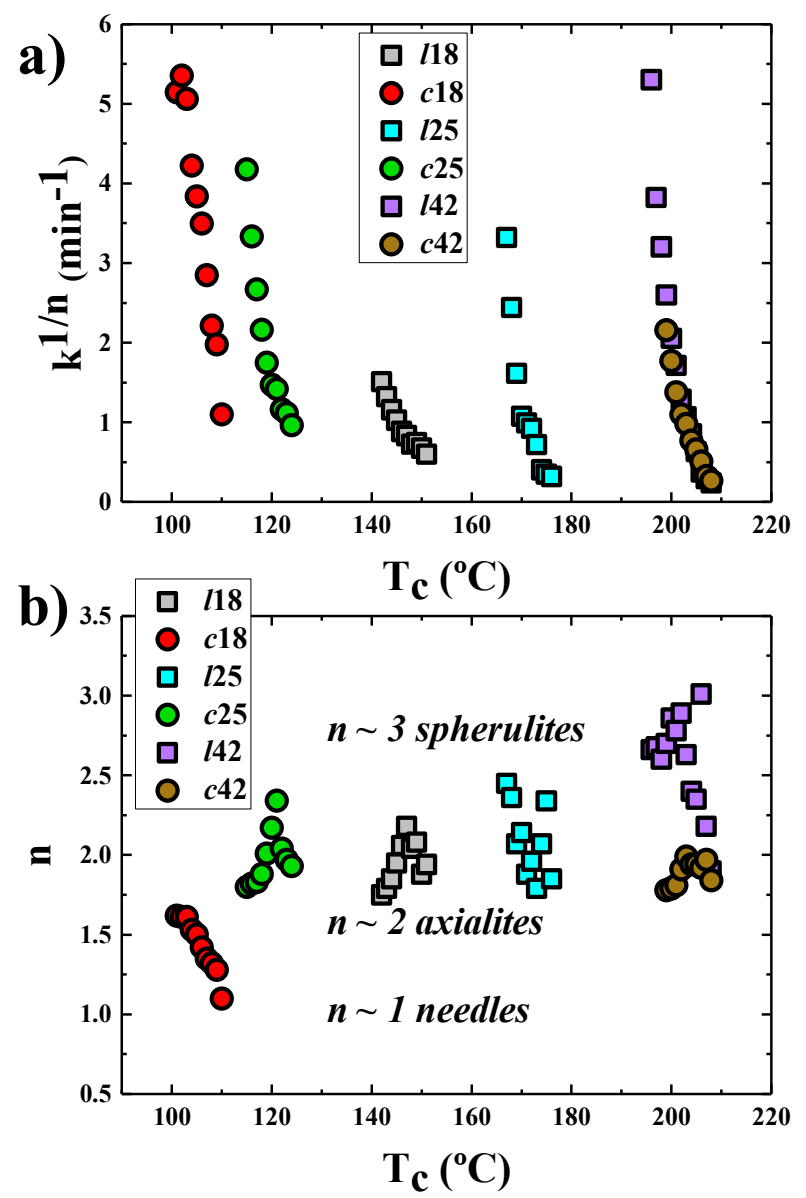

Figure 5. a) Normalized rate crystallization constant $\left(k^{1 / n}\right)$ of the Avrami model as a function of $T_{c}$ and b) Avrami index as a function of $T_{c}$ for linear and cyclic P3HT.

The values observed for $k^{1 / n}$ are proportional to the overall crystallization rate. This is an excellent way to compare experimental data points (experimental values of $1 / \tau_{50 \%}$ in Figure $3 a$ ) with the Avrami theory parameters (calculated $k^{1 / n}$ values, plotted as data points in Figure 5a). The remarkable qualitative similarity between Figure $3 a$ and Figure $5 a$ is a consequence of the excellent fit of the Avrami equation to the overall crystallization rate data.

The values of the Avrami index fluctuate between 1.25 and 3, depending on the sample, but almost all of them are around 2. In polymers, the possible dimensions of the growing crystals are 2 or 3 , corresponding to axialites (two dimensional lamellar aggregates) and spherulites (superstructural three dimensional aggregates) nucleated instantaneously, respectively. It seems that the cyclic samples have lower Avrami indexes as compared to their linear counterparts. As will be demonstrated below, the results are reasonable on the basis of the observed superstructural morphologies.

Texture and morphological experimental observations were performed by Polarized Light Optical Microscopy (PLOM). The superstructural morphology at the microscopic scale of both linear and cyclic P3HT polymers was observed by PLOM.

In the case of linear P3HT samples, the observed superstructural morphology was very small, as the nucleation density that developed upon cooling was extremely high. When the nucleation density is high, a microspherulitic or micro-axialitic morphology is usually developed, as superstructures can impinged on one another even before a three dimensional structure can be formed. These observations for linear P3HT are consistent with the results obtained by crystallization kinetics, where the Avrami index for linear P3HT varied between 2 and 3.

In Figure 6, 125 and c25 samples are compared and the different morphologies observed after an isothermal crystallization process. Both samples were crystallized isothermally at temperatures close 
(above) to each crystallization peaks (observed by DSC before). The samples observed in Figure 6a and 6b

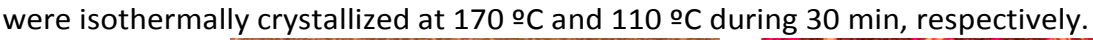
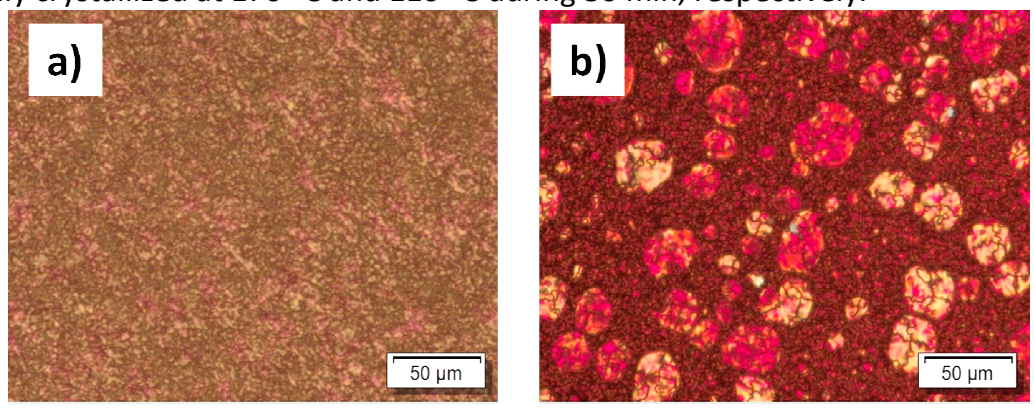

Figure 6. PLOM micrographs revealing the optical properties of both a) $125-\mathrm{P} 3 \mathrm{HT}$ and b) $\mathrm{c} 25-\mathrm{P} 3 \mathrm{HT}$ samples crystallized isothermally at $170 \circ \mathrm{C}$ and at $110 \circ \mathrm{C}$, respectively.

For the linear sample, it is possible to see a homogeneous texture over the whole surface where P3HT superstructural aggregates are observed, just as described above. The optical properties observed for the cyclic P3HT samples are very different as compared with their linear analogues.

For the cyclic sample, Figure 6 shows two well defined crystalline textures. First, some large distorted roselike objects, some of which display Maltese crosses and surrounding these textures, there is a background with very small birrefringent points that resembled the microscopic superstructural crystalline texture observed for the linear P3HT crystals sample.

The topology effect induces remarkable changes in the crystalline texture of the sample. Due to these notable changes, isothermal crystallization experiments at different temperatures were performed in order to corroborate these large changes in the morphology. For cyclic samples, experiments at high temperatures were done and different textures were observed even well above their calorimetric melting points. Isothermal crystallization experiments were done for 18, 25 and 42 cyclic P3HT samples at 190, 175 and 200 ㅇ C during 10 and $20 \mathrm{~min}$, respectively. Figure 7 shows the morphologies observed for $c 18, c 25$ and c42 P3HT samples.

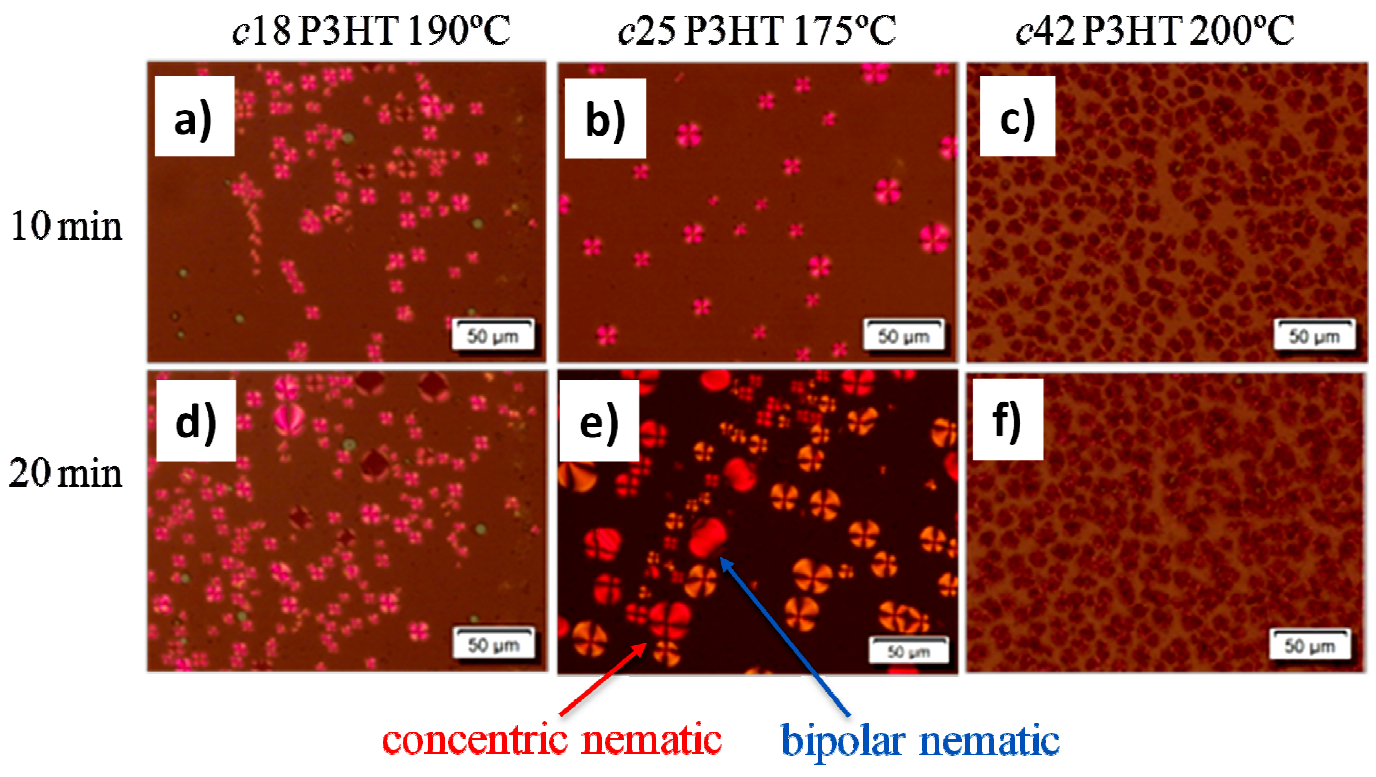

Figure 7. PLOM micrographs of isothermally crystallized cyclic P3HT samples of a) $c 18$ sample crystallized at 190 ㅇ C during $10 \mathrm{~min}$, b) c25 sample crystallized at 175 o C during $10 \mathrm{~min}$, c) c42 sample crystallized at 200ㄷ during $10 \mathrm{~min}$, and d), e), f) same samples crystallized at the indicated temperatures during $20 \mathrm{~min}$.

These images reveal significant changes in the phase behavior of cyclic $\mathrm{P} 3 \mathrm{HT}$ samples as compared to their linear analogues. The observed textures correspond to bipolar and concentric nematic droplets as Fernandez-Nieves et al. demonstrated in their articles. ${ }^{30,31}$ They studied some nematic liquid crystals in 
confinement state and when they started cooling the samples from the isotropic state, the samples started forming concentric nematic droplets and after some time, they changed to bipolar droplets. The interpretation of the authors implies that the droplets changes periodically between concentric and bipolar configurations.

In our case, after the cooling process, the observed droplets initially form an scaped concentric nematic texture (Figure $7 \mathrm{~b}$ ) and then undergo a transient regime, in which defects are created and finally they revert to the lowest energy nematic bipolar structure (Figure 7e). Coulembier et al. demonstrated in this type of macrocyclic P3HT samples forms a tubular assembly, with an estimated diameter of $6+/-3 \mathrm{~nm}$. ${ }^{32}$ These confined rodlike molecules display orientational order along an average direction and are typically formed by nematic liquid crystals.

The optical properties of liquid crystal phases often directly reflect the symmetry of their structures. The birefringence, or anisotropy of the refractive index along two directions, is one of characteristic physical properties of liquid crystals, and it allows the visualization of the macroscopic molecular orientation. In thin liquid crystal samples placed between two crossed polarizers under an optical microscope, various textures and birefringence colors will be observed, as are shown in Figure 7. These textures and colors not only look beautiful but also contain information about the macroscopic structure of the LC phases. Change in the droplet morphologies due to the change in the polymer molar masses values and rate of cooling from an isotropic state have also been reported in different polymer dispersed liquid crystals. ${ }^{33,34}$ In the case of the c18-P3HT sample, it is possible to observe that when the cooling conditions changes (high cooling rate) for example, the droplets morphology also changes, going from almost concentric nematic droplets (Figure 7a and $7 \mathrm{~b}$ ) to all bipolar nematic droplets, as observed in Figure $8 \mathrm{~b}$.

The different PLOM images correspond to different temperatures chosen during the cooling scan performed in the microscope. First, the sample was heated until $250 \stackrel{\circ}{ } \mathrm{C}$, where the samples reached the isotropic state as we have observed before by DSC experiments, and then the sample was cooled down at a high cooling rate (i.e., $50 \stackrel{\circ}{\circ} / \mathrm{min}$ ). In Figures $8 \mathrm{a}$ and $8 \mathrm{~b}$, bipolar nematic droplets are observed at temperatures higher than the crystallization exotherm observed in the DSC for c18 P3HT. Upon further cooling, in Figures $8 \mathrm{c}$ and $8 \mathrm{~d}$, at temperatures lower than the $T_{c}$ observed by DSC, the images show a solidification of the droplets formed. Due to the high cooling rate and the low crystallinity of this sample, the $\mathrm{P} 3 \mathrm{HT}$ is not able to crystallize in the background surrounding the nematic droplets, as it happened in Figure $4 \mathrm{~b}$ for example.

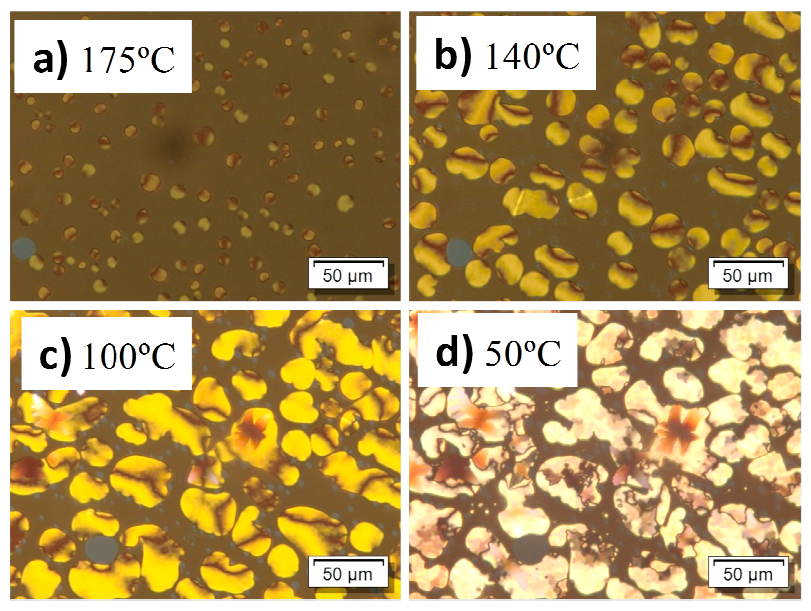

Figure 8. PLOM micrographs of non-isothermally crystallized c18 P3HT samples. Images are taken at

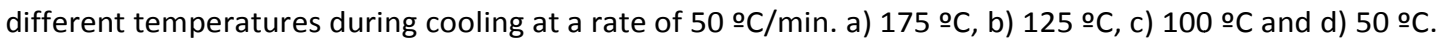

In order to confirm that the observed bipolar droplets texture represents a nematic liquid crystalline (LC) phase, WAXS experiments were performed, attempting to detect a nematic-periodicity peak. Nematic liquid crystalline phases have long range orientational order but no positional order. Figure 9 shows WAXS scattering profiles of the studied linear and cyclic P3HT samples measured at room temperature. 


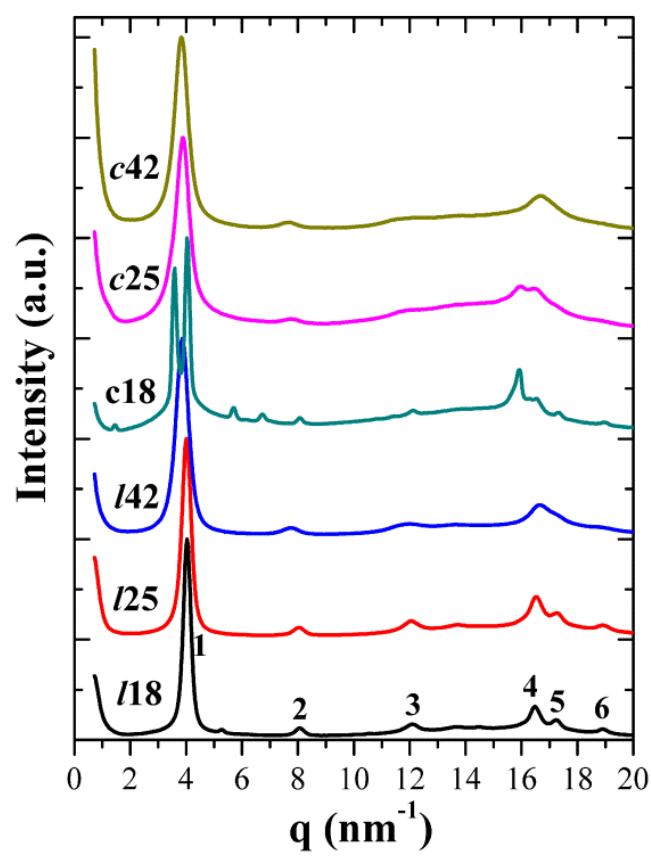

Figure 9. WAXS diffractograms for the indicated linear and cyclic P3HT samples at RT. P3HT diffraction peaks are labelled according to reference 16.

The first three peaks (1-3) shown in the WAXS profile for $/ 18$ and $/ 25$ can be identified as series of (h00) reflections. Peak 4 is a superposition of the $(020)$ and a weak (002) reflection. The last two reflections can be attributed to the plane indices (120) and (220). ${ }^{16}$ For 142, the peaks 4, 5, and 6 are less well resolved. As for the cyclic $\mathrm{P} 3 \mathrm{HT}$, great differences are observed for $\mathrm{c} 18$. Two peaks exist at a similar q value for the (100) peak of I18. Several new peaks are found. The diffraction features of c25 and c42 look similar to that of 142. Figure 10 shows a detailed analysis of c18. Those peaks labelled with Arabic numbers represent a similar structure as the $/ 18$ shown in Figure 9.

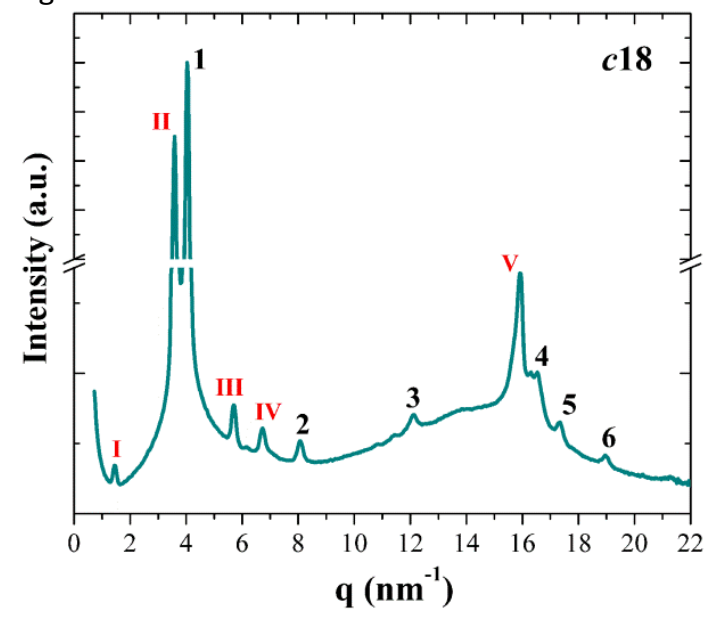

Figure 10. WAXS diffractograms of $c 18$ with all the peaks labelled.

Those peaks correspond to an orthorhombic unit cell structure with the following cell parameters: $a=1.55$ $\mathrm{nm}, \mathrm{b}=0.76 \mathrm{~nm}, \mathrm{c}=0.76 \mathrm{~nm}, \alpha=\beta=\gamma=90$ ( (form 1), which agrees with the normal linear P3HT (ref. 16). New peaks are labelled as Roman numbers. Those new sharp peaks apparently do not agree with the feature of a nematic phase but probably indicate a new crystal form. Peak I with a remarkable lattice spacing of $4.39 \mathrm{~nm}$, roughly agrees with the diameter of the ring. There is no other possible characteristic structure in the system that exhibits such a large length. Peak II has a lattice spacing of $1.75 \mathrm{~nm}$, larger than normal $d_{100}$ of linear P3HT $(1.57 \mathrm{~nm}$ ref 16 , and $1.56 \mathrm{~nm}$ in 118$)$ which should correspond to a different packing also along the alkyl chain direction. Two additional peaks in between peak 1 (100) and peak 2 (200) appear in c18. Interestingly, a relationship among the peaks II, III, and IV is found: 


$$
d_{I V}=\frac{d_{I I} * d_{I I I}}{\sqrt{d_{I I}^{2}+d_{I I I}^{2}}}
$$

This relationship indicates a possible assignment of peaks II, III, and IV as the 100, 010, and 110, respectively, with a-axis orthogonal with the b-axis. Peak $V$ with a lattice spacing of $0.39 \mathrm{~nm}$ is better assigned to the (002) reflection. A possible unit cell dimension for this new orthorhombic crystal structure thus can be deduced as: $a=1.75 \mathrm{~nm}, b=1.10 \mathrm{~nm}, c=0.78 \mathrm{~nm}, \alpha=\beta=\gamma=90^{\circ}$ (new form). We have to note that the peak assignment is not rigorous because of the limited number of reflections available. All the above mentioned reflections are summarized in Table 1.

Table 1. Diffraction peaks, lattice spacings and indexing of the WAXS of $c 18$.

\begin{tabular}{|c|c|c|c|c|}
\hline & $\mathrm{q}\left(\mathrm{nm}^{-1)}\right.$ & $\mathrm{d}(\mathrm{nm})$ & $\begin{array}{c}\text { Form I } \\
(\mathrm{hkl})\end{array}$ & $\begin{array}{c}\text { New Form } \\
(\mathrm{hkl})\end{array}$ \\
\hline $\mathrm{I}$ & 1.465 & 4.29 & & $\begin{array}{c}\text { diameter of } \\
\text { the ring }\end{array}$ \\
\hline $\mathrm{II}$ & 3.582 & 1.75 & & 100 \\
\hline 1 & 4.052 & 1.55 & 100 & \\
\hline $\mathrm{III}$ & 5.706 & 1.10 & & 010 \\
\hline $\mathrm{IV}$ & 6.724 & 0.93 & & 110 \\
\hline 2 & 8.071 & 0.79 & 200 & \\
\hline 3 & 12.08 & 0.52 & 300 & \\
\hline $\mathrm{V}$ & 15.92 & 0.39 & & 002 \\
\hline 4 & 16.52 & 0.38 & $020 / 002$ & \\
\hline 5 & 17.33 & 0.36 & 120 & \\
\hline 6 & 18.96 & 0.33 & 220 & \\
\hline
\end{tabular}

Variable temperature WAXS was carried out on c25 to observe the signature of nematic phase at elevated temperatures as shown in Figure 11. It is interesting to see new peaks appear when the temperature increases to $100^{\circ} \mathrm{C}$. The positions of the peaks are similar to those of $c 18$. The only exception is that peak I at the lowest $q$ is missing, which can be explained by the fact that the diameter of the ring of c 25 exceeds the limit of detection. The new peaks vanish, together with the peaks at $\sim 16 \mathrm{~nm}^{-1}$, at $155^{\circ} \mathrm{C}$. It seems that only the order along side chain packing remains at this temperature. This feature looks very similar to a nematic phase of a liquid crystal. The sample becomes amorphous at $170^{\circ} \mathrm{C}$. The new diffraction peaks are not observed in c42. It is anticipated that larger rings behave closer to its linear analogs. Another possible reason is that c42 contains a larger fraction of linear impurities. 


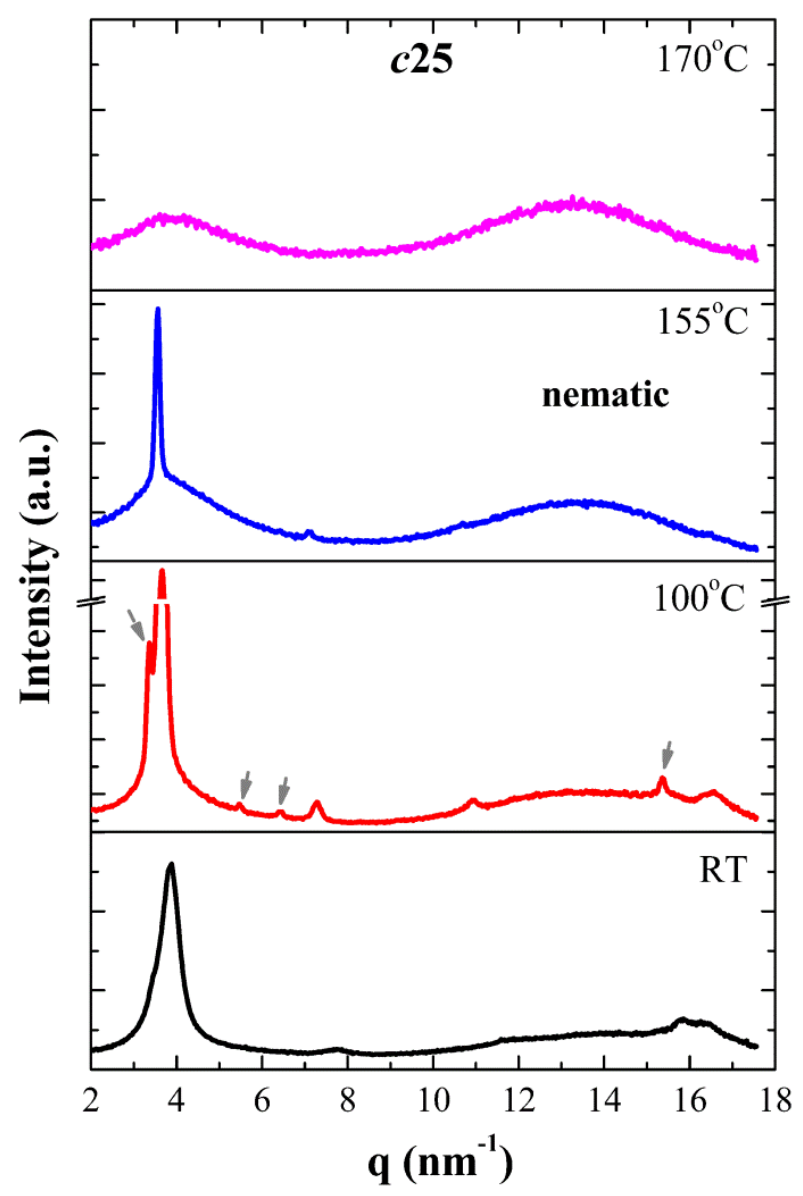

Figure 11. WAXS diffractograms of $c 25$ at different temperatures. Arrows show the new peaks that appear when the sample is heated to $100^{\circ} \mathrm{C}$.

We are interested in how the two crystal forms correlate with cyclic topology. If we imagine the interchain packing of a cyclic $\mathrm{P} 3 \mathrm{HT}$, two possibilities may exist. The first case is that the cyclic molecule plane is perpendicular to the thiophene ring plane, as illustrated in Figure 12. Intra-ring $\pi-\pi$ packing and inter-ring $\pi-\pi$ packing organized alternatively. This crystal structure is basically the same as in the linear P3HT, which is called form I in this work. Intuitively, this type of packing will become difficult and unstable for small rings because of the free energy penalty raised by the folding. In fact, a "planar conformation" of the thiophene ring would be favored (as will be shown by the DFT calculation in the following section). In this case, all the side chains have to be pointing "outwards" to avoid crowding. This will naturally result in a large degree of torsion, which renders perfect planar packing of main chain thiophenes. As shown in Figure 12, under this circumstance, the a-axis would be longer because it must include an additional thiophene unit, and the baxis would be longer because of the torsion of thiophene units. However, the c-axis should be similar to that of form I.

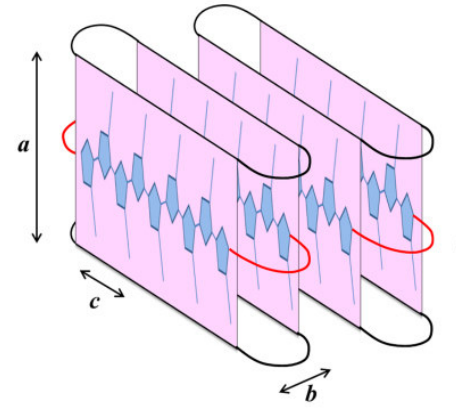

form I

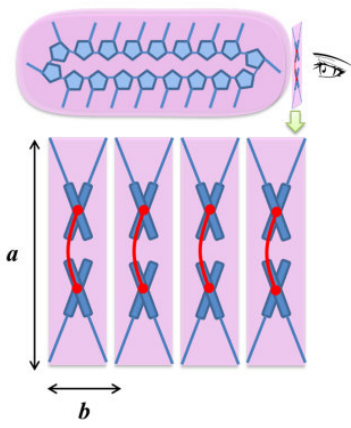

new form

Figure 12. Schematic of the two possible packing of cyclic $\mathrm{P} 3 \mathrm{HT}$, which results in two different crystal forms. 
Ding et al. ${ }^{35}$ observed in Poly(butylene naphthalate) samples similar reflections at low q-range that they attributed to the existence of a smectic liquid-crystalline glass at room temperature, possessing a characteristic Schlieren texture when observed by PLOM experiments.

In order to confirm that an isotropic-nematic (IN) transition was indeed observed by PLOM and WAXS experiments, the PLOM-S technique was employed and compared with DSC results, as some small peaks were observed at high temperatures in the cyclic samples by DSC.

As an example, the results collected for $/ 25$ and $c 25$ P3HT samples are shown in Figure 13.

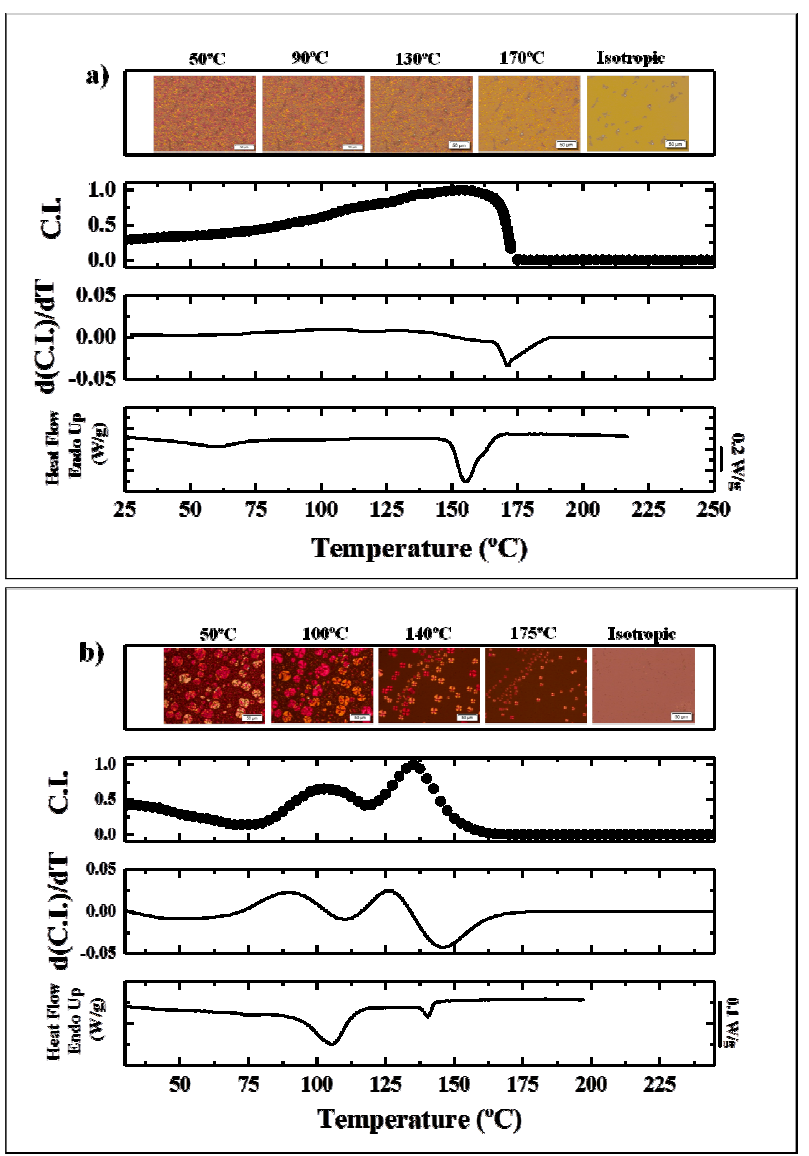

Figure 13. PLOM-S measurements of the isotropic-nematic (IN) transition for a) $/ 25$ P3HT sample and b) c25 P3HT sample. In the top part of each figure, PLOM images are shown upon cooling from the isotropic state to room temperature at $5 \mathrm{o} / \mathrm{min}$. The temperatures at which the images were acquired are indicated above the micrographs. In the second part of the figures (just below the PLOM micrographs), the evolution of the conversion index (C.I., defined as the normalized integral value of the transmitted light between 580 and $700 \mathrm{~nm}$ ) is plotted versus temperature. Third, $\mathrm{d}\left(\mathrm{C} . \mathrm{I}\right.$.)/dT from which the $T_{\mathrm{IN}}$ are determined as the peak temperatures. Finally, in the bottom part of the figures, the DSC cooling scans obtained are plotted, in order to compare the transitions observed by the two different techniques.

The procedure used was the same for all samples and the rest of the results are shown in the supporting information (Figure S5). Moreover, the relative transmittance spectra (relative transmission vs wavelength, $\lambda$ ) recorded at different temperatures during the phase transitions for all the P3HT samples are also shown in the supporting information of this manuscript (Figure S6).

The relative transmission spectra (Figure S6) were then integrated between 580 and $700 \mathrm{~nm}$ and the resulting value normalized between 1 and 0 to obtain the conversion index, C.I., of the phase transformation. The IN transition when plotted against temperature (d(C.I.)/dT), exhibit a maximum at the temperature at which the phase transformation occurs more rapidly. In order to analyze and demonstrate the different transitions observed during the cooling scans, this method is compared with DSC results.

As can be seen in Figure 13, for the linear 125 P3HT sample, only one main and sharp peak is observed in the $\mathrm{d}(\mathrm{C} . \mathrm{I}$.)/dT plot, that corresponds to the crystallization exotherm or peak at 155 o $\mathrm{C}$, observed during the DSC cooling run. On the other hand, for cyclic c25 P3HT sample, two main peaks are observed in the $\mathrm{d}(\mathrm{C} . \mathrm{I}.) / \mathrm{dT}$ plot, where the second peak at lower temperatures corresponds with the $T_{c}$ value at $105 \stackrel{\circ}{ } \mathrm{C}$ 
observed during the DSC cooling run. The first peak at $140{ }^{\circ} \mathrm{C}$ that can be observed during the PLOM-S experiment, corresponds to the IN transition, where the bipolar concentric nematic droplets are formed as confirmed by means of the PLOM micrographs, DSC scans and WAXS diffractograms shown here. To further compare our experimental studies with theoretical works, we have obtained the IN transition temperatures for cyclic $\mathrm{P} 3 \mathrm{HT}$ samples at the same range that Zhang et al. ${ }^{36}$ predicted combining self-consistent field theory (SCFT) with atomistic simulations of semiflexible chains under tension, to estimate the nematic coupling constant $\alpha$ for semiflexible polymers. They directly estimate $T_{\mathrm{IN}}$ for P3HT with various chain lengths $S$, given by $T_{\mathrm{IN}}(S)=535 \mathrm{~K}(1-1.64 / \mathrm{S})$. Using the estimated $T_{\mathrm{IN}}$, they predict that P3HT is nematic above the reported crystal melting temperatures as we demonstrate experimentally by cyclic $\mathrm{P} 3 \mathrm{HT}$ samples of different chain lengths.

Finally, we have performed density functional theory (DFT) calculations in cyclic and linear P3HT structures including 9 hexyl-substituted thiophene rings. The optimized structures are collected in Figure 14.
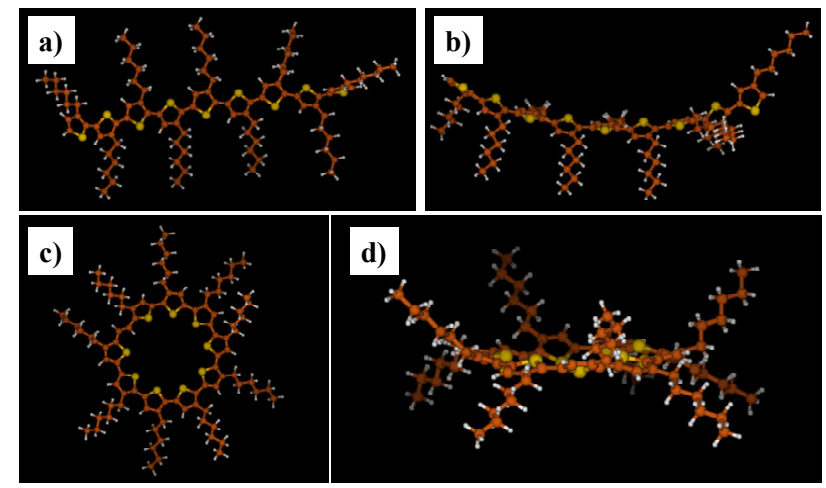

Figure 14. Molecular structures for the linear $(a, b)$ and cyclic (c,d) P3HT structures, observed from the top $(a, c)$ or from the side $(b, d)$ view.

After cyclization, the strain induced by forcing these molecules to adopt a cyclic conformation leads to more rigid cyclic chains as compared to linear $\mathrm{P} 3 \mathrm{HT}$, especially at low molecular weights. Inspecting the molecular structures, the interaction among cyclic structures is expected to be less effective than in the linear ones due to the sterical hindrance exerted by the hexyl groups, pending from both sides of the "planar" ring (see Figure 14, panel d), while in the linear structure, they remain in the same side (see Figure 14 , panel b), allowing the thiophene rings to interact with other chains. The ring conformation in Figure 14d agrees well with the unit cell packing we proposed in Figure 12.

\section{Conclusions}

Our study was motivated by the different behaviors observed in the literature when chain topology is changed from linear to cyclic. Different trends have been reported for different polymeric chains, but so far no study has focused on P3HT chains. Cyclic topology induces dramatic changes in P3HT polymer conformation and chain packing. The macrocycles are more restricted in the crystalline $\mathrm{P} 3 \mathrm{HT}$ phase and $T_{c}$, $T_{m}$ and $\Delta H$ decreased in comparison to those measured in linear analogues. PLOM experiments revealed the formation of Liquid Crystalline bipolar and concentric droplets at temperatures before crystal formation for cyclic P3HT only. Detailed WAXS analysis showed that two crystalline structures were formed for c18 and c25, corresponding to two possible conformations of the polymer caused by the cyclic topology. A nematic-periodicity was observed at elevated temperatures in the P3HT cyclic samples due to the long-range orientational order of the molecular axes and PLOM/Spectroscopy results demonstrated that the nematic droplets are formed when the samples are cooled down from an isotropic state to the crystalline state. These isotropic-nematic IN transition temperatures are above the crystal melting temperatures as are confirmed by results obtained by correlating PLOM, Spectroscopy and DSC techniques. The changes induced in $\mathrm{P} 3 \mathrm{HT}$ by cyclic topologies in low molecular weight chains, such as those employed in this work, can be attributed to the more rigid character of cyclic chains as compared to linear ones, as ascertained by density functional theory (DFT) calculations. DFT results also confirm the unique planar conformation of cyclic P3HT, which agrees with the WAXS results. Such increased rigidity is responsible for the difficulties experienced by cyclic P3HT in crystallizing from the melt and for the development of Liquid Crystalline phases prior to crystallization. 


\section{Conflicts of interest}

There are no conflicts to declare.

\section{Acknowledgements}

J.M. acknowledges support from the Provincial Council of Gipuzkoa under the program Fellow Gipuzkoa and "Fomento San Sebastián" in the framework program "Retorno del Talento Local" Donostia up! 2016. We acknowledge the help of Dr. Jaime Martín and Ms. Sara Marina with the combined Microscopy/Spectroscopy measurements. J.M. and A.J.M. acknowledge funding by Mineco MAT201783014-C2-1-P project. N.D. and O.C. are grateful to the Science Policy Office of the Belgian Federal Government (PAI 7/5) and to the Belgian FRFC-FNRS ( $n^{\circ} 2.4508 .12$ ). O.C. is Research Associates of the F.R.S.-FNRS. G.L. is grateful to the Youth Innovation Promotion Association of the Chinese Academy of Sciences (2015026). D.W. and A.J.M acknowledge the support from the National Natural Science Foundation of China (51820105005). All authors gratefully acknowledge the support of the EU through the H2020-MSCA-RISE-2017-778092 BIODEST project. Technical and human support provided by IZO-SGI, SGIker (UPV/EHU, MICINN, GV/EJ, ERDF and ESF) is gratefully acknowledged for assistance and generous allocation of computational resources.

\section{References}

1. A. T. Lorenzo, M. L. Arnal, J. Albuerne and A. J. Müller, Polym. Test., 2007, 26, 222-231.

2. R. A. Pérez-Camargo, A. Mugica, M. Zubitur and A. J. Müller, in Polymer Crystallization I: From Chain Microstructure to Processing, eds. F. Auriemma, G. C. Alfonso and C. de Rosa, Springer International Publishing, Cham, 2017, DOI: 10.1007/12_2015_326, pp. 93-132.

3. M. E. Córdova, A. T. Lorenzo, A. J. Müller, J. N. Hoskins and S. M. Grayson, Macromolecules, 2011, 44, 1742-1746.

4. N. Zaldua, R. Liénard, T. Josse, M. Zubitur, A. Mugica, A. Iturrospe, A. Arbe, J. De Winter, O. Coulembier and A. J. Müller, Macromolecules, 2018, 51, 1718-1732.

5. H.-H. Su, H.-L. Chen, A. Díaz, M. T. Casas, J. Puiggalí, J. N. Hoskins, S. M. Grayson, R. A. Pérez and A. J. Müller, Polymer, 2013, 54, 846-859.

6. R. A. Pérez, M. E. Córdova, J. V. López, J. N. Hoskins, B. Zhang, S. M. Grayson and A. J. Müller, React. Funct. Polym., 2014, 80, 71-82.

7. J. V. López, R. A. Pérez-Camargo, B. Zhang, S. M. Grayson and A. J. Müller, RSC Adv., 2016, 6, 48049-48063.

8. Y. Tezuka, Ohtsuka, T. , Adachi, K. , Komiya, R. , Ohno, N. and Okui, N. , Macromol. Rapid Commun. 2008, 29, 1237-1241.

9. H. Takeshita, M. Poovarodom, T. Kiya, F. Arai, K. Takenaka, M. Miya and T. Shiomi, Polymer, 2012, 53, 5375-5384.

10. M. Mayor and C. Didschies, Angew. Chem., Int. Ed., 2003, 42, 3176-3179.

11. M. Ball, Y. Zhong, B. Fowler, B. Zhang, P. Li, G. Etkin, D. W. Paley, J. Decatur, A. K. Dalsania, H. Li, S. Xiao, F. $\mathrm{Ng}$, M. L. Steigerwald and C. Nuckolls, J. Am. Chem. Soc. 2016, 138, 12861-12867.

12. M. lyoda and H. Shimizu, Chem. Soc. Rev., 2015, 44, 6411-6424.

13. S. Chandrasekhar, B. K. Sadashiva and K. A. Suresh, Pramana, 1977, 9, 471-480.

14. V. K. Thakur and M. R. Kessler, Liquid Crystalline Polymers, Springer International Publishing 2015.

15. A. Selevou, G. Papamokos, M. Steinhart and G. Floudas, The J. Phys. Chem. B, 2017, 121, 7382-7394.

16. Z. Wu, A. Petzold, T. Henze, T. Thurn-Albrecht, R. H. Lohwasser, M. Sommer and M. Thelakkat, Macromolecules, 2010, 43, 4646-4653.

17. N. Delbosc, J. De Winter, S. Moins, A. Persoons, P. Dubois and O. Coulembier, Macromolecules, 2017, 50, 1939-1949.

18. J. Martin, E. C. Davidson, C. Greco, W. Xu, J. H. Bannock, A. Agirre, J. de Mello, R. A. Segalman, N. Stingelin and K. C. Daoulas, Chem. Mater., 2018, 30, 748-761.

19. M. Frisch, G. Trucks, H. Schlegel, G. Scuseria, M. Robb, J. Cheeseman, G. Scalmani, V. Barone, G. Petersson and H. Nakatsuji, Gaussian 16, revision A.03; 2016.

20. C. Lee, W. Yang and R. G. Parr, Phys. Rev. B, 1988, 37, 785.

21. A. D. Becke, J. Chem. Phys., 1993, 98, 5648-5652.

22. W. J. Hehre, R. Ditchfield and J. A. Pople, The J. Chem. Phys., 1972, 56, 2257-2261.

23. D. G. Tsalikis, P. V. Alatas, L. D. Peristeras and V. G. Mavrantzas, ACS Macro Lett., 2018, 7, 916-920.

24. H. Xiao, C. Luo, D. Yan and J.-U. Sommer, Macromolecules, 2017, 50, 9796-9806. 
25. K. Iyer and M. Muthukumar, J. Chem. Phys., 2018, 148, 244904.

26. V. Arrighi, S. Gagliardi, F. Ganazzoli, J. S. Higgins, G. Raffaini, J. Tanchawanich, J. Taylor and M. T. F. Telling, Macromolecules, 2018, 51, 7209-7223.

27. L. Mandelkern, Crystallization of Polymers: Volume 1: Equilibrium Concepts, Cambridge University Press, Cambridge, 2 edn., 2002.

28. G. Strobl, in Progress in Understanding of Polymer Crystallization, eds. G. Reiter and G. R. Strobl, Springer Berlin Heidelberg, Berlin, Heidelberg, 2007, DOI: 10.1007/3-540-47307-6_23, pp. 481-502.

29. M. J. Avrami, J. Chem. Phys., 1941, 9, 177-184.

30. A. Fernández-Nieves, D. R. Link, M. Márquez and D. A. Weitz, Phys. Rev. Lett., 2007, 98, 087801.

31. T. Lopez-Leon, A. Fernandez-Nieves, M. Nobili and C. Blanc, Phys. Rev. Lett., 2011, 106, 247802.

32. O. Coulembier, G. Deshayes, M. Surin, J. De Winter, F. Boon, C. Delcourt, P. Leclère, R. Lazzaroni, P. Gerbaux and P. Dubois, Polym. Chem., 2013, 4, 237-241.

33. J. K. Srivastava, R. K. Singh, R. Dhar and S. Singh, Liq. Cryst., 2011, 38, 849-859.

34. J. K. Srivastava, R. K. Singh, R. Dhar and S. Singh, Liq. Cryst., 2012, 39, 1402-1413.

35. Q. Ding, D. Jehnichen, M. Göbel, M. Soccio, N. Lotti, D. Cavallo and R. Androsch, Eur. Polym. J., 2018, 101, 90-95.

36. W. Zhang, E. D. Gomez and S. T. Milner, Macromolecules, 2015, 48, 1454-1462. 